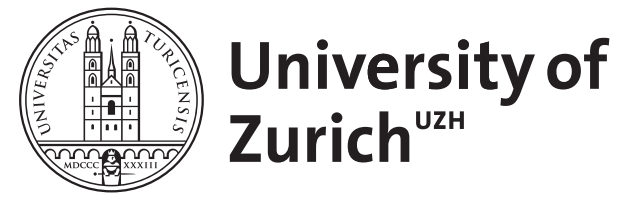

\title{
Unterstützung der politischen Kommunikation
}

\author{
Schwabe, Gerhard
}

DOI: https://doi.org/10.1007/3-540-46272-4_27

Posted at the Zurich Open Repository and Archive, University of Zurich

ZORA URL: https://doi.org/10.5167/uzh-57207

Book Section

Accepted Version

Originally published at:

Schwabe, Gerhard (2006). Unterstützung der politischen Kommunikation. In: Kröger, Detlef; Wind, Martin. Handbuch IT in der Verwaltung. Berlin / Heidelberg, Deutschland: Springer, 685-705.

DOI: https://doi.org/10.1007/3-540-46272-4_27 


\title{
Unterstützung der politischen Kommunikation
}

\author{
Prof. Dr. Gerhard Schwabe, Universität Zürich
}

\section{Einleitung}

Weite Bereiche der politischen Kommunikation wurden lange Zeit als nicht durch moderne Informations- und Kommunikationstechnik unterstützbar angesehen. Zwar konnte man sich gut vorstellen, dass sich die Politik im Internet präsentieren könnte und im Rahmen der Diskussion zur elektronischen Bürgerinformation (Lenk et al 1990) wurde schon früh auf das Potential von zuerst BTX und dann dem Internet hingewiesen (Schwabe 1996; Schwabe et al. 1997), um Bürger nicht nur besser zu informieren, sondern auch sie besser an der politischen Willensbildung zu beteiligen. Die Hauptträger der politischen Kommunikation - die Politiker - wurden aber bei dieser Diskussion weitgehend aussen vor gelassen. Auch bei der wachsenden IT-Unterstützung von Verwaltungen im Rahmen der E-Government-Diskussion steht die Politik nicht im Zentrum. Dies zu Unrecht, denn eine leistungsfähige Politik ist für den Standort Deutschland wesentlich und der Unterstützungsbedarf sowie die Offenheit der Politiker wird regelmäßig unterschätzt. Im Pilotprojekt Cuparla (Schwabe 2000) konnte in der zweiten Hälfte der 90er Jahre gezeigt werden, wie eine Unterstützung für die Kommunalpolitik aussehen kann (vgl. Kapitel 4.2). Die in Stuttgart eingeführte Software wurde in der Zwischenzeit weiterentwickelt (Krcmar et al 2002; Schwabe 2003) und ist immer noch im Einsatz. Auch andere Großstädte haben inzwischen ähnliche Systeme im Einsatz. Trotz dieser erfreulichen Fortschritte sind bisher nur einzelne Schritte im Politikprozess umfassend unterstützt.

Abbildung 1 zeigt die einzelnen Schritte der entscheidungsorientierten, politischen Arbeit in einer Kommune. Dabei sind die einzelnen Prozessschritte umso dunkler eingefärbt, je umfassender die in ihnen stattfindende Kommunikation und Arbeit mit IT unterstützt ist.

\section{Politikprozess}

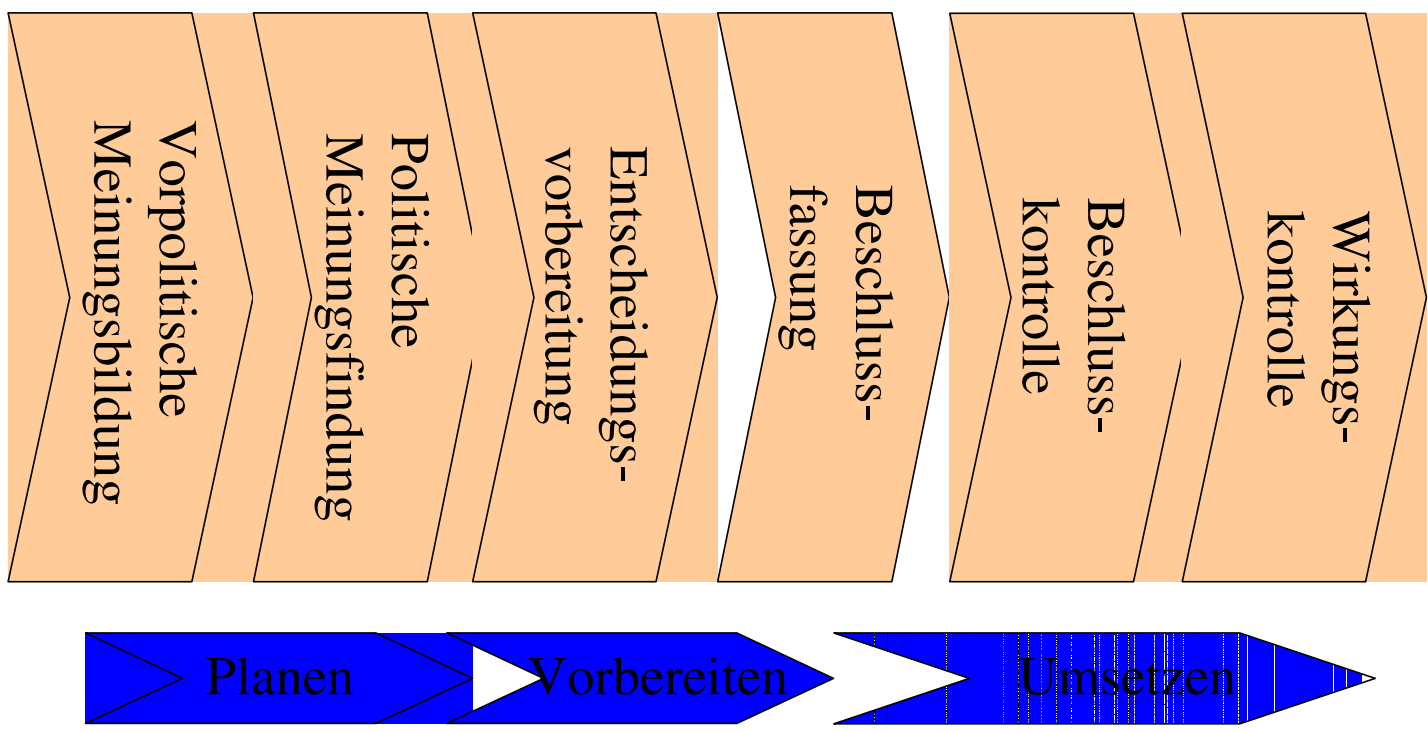

Begleitender Verwaltungsprozess

\section{Abbildung 1: Der Politikprozess}

Die eigentliche Beschlussfassung ist vergleichsweise am umfassendsten unterstützt. Innerhalb der Verwaltung wird sie mit kommunalen Sitzungsdiensten (vgl. Kapitel 3.1) vorbereitet und Ratsinformationssysteme (vgl. Kapitel 3.2) stellen den gewählten Vertretern die Beschluss- 
vorlagen zur Verfügung. Für die Kommunikation zur eigenen politischen Meinungsbildung und Entscheidungsvorbereitung stehen den Räten Ratskommunikationssysteme und Bürosysteme (vgl. Kapitel 4) sowie Ratskoordinations- und Ratskooperationssysteme zur Verfügung. Es sind erste Ansätze von Systemen sichtbar, welche die Beschlusskontrolle und die Wirkungskontrolle in kommunale Führungsinformationssysteme (vgl. Kapitel 5 und 6) aufnehmen. Für die Wirkungskontrolle, wie auch für die vorpolitische Meinungsbildung, ist die Unterstützung der Kommunikation mit dem Bürger (vgl. Kapitel 7) hilfreich.

Bevor auf die einzelnen Unterstützungsansätze eingegangen wird, wird im folgenden kurz aufgezeigt, in welchem Kommunikationsnetz ein Rat typischerweise eingebunden ist. Bei der Diskussion einzelner Unterstützungsansätze werden jeweils zuerst der Unterstützungsansatz und die zugrundeliegenden IT-Systeme vorgestellt, dann werden auf Beispiele aus der Praxis zurückgegriffen . Diese beziehen sich zu einem grossen Teil auf die Landeshauptstadt Stuttgart.

\section{Kommunikation in der kommunalen Politik}

Der einzelne Rat ist für seine Gemeinderatsarbeit in ein umfassendes Netzwerk von Kommunikationsbeziehungen eingebettet. Diese lassen sich den drei Bereichen "Gemeinderat", "Verwaltung" und "Umfeld" zuordnen (vgl Abbildung 2).

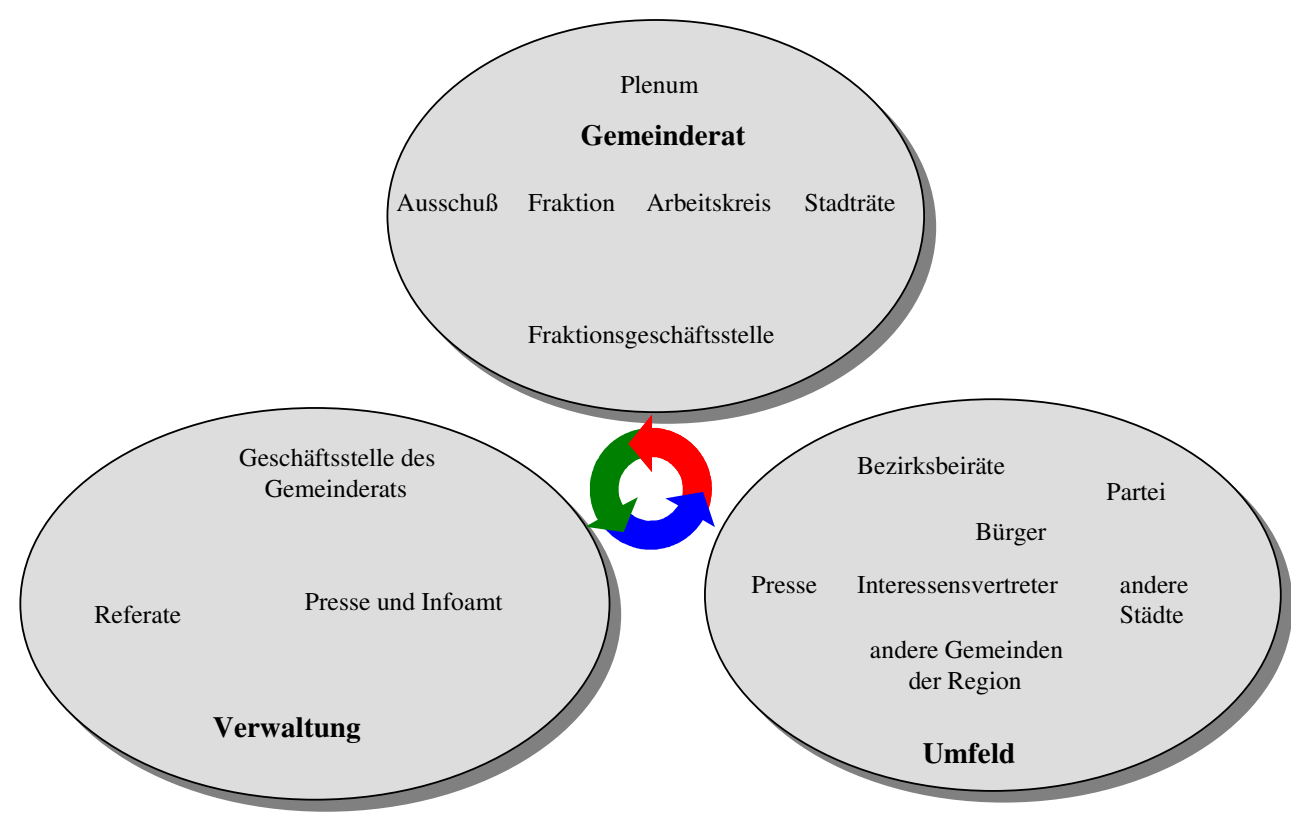

Abbildung 2: Kooperationspartner der Politik (Bsp. Gemeinderat)

Innerhalb des Gemeinderats arbeitet das einzelne Ratsmitglied im Plenum, in einzelnen Ausschüssen, in Arbeitskreisen und mit einzelnen anderen Stadträten zusammen. Die Fraktionsgeschäftstelle ist eine Schnittstelle sowohl zur Verwaltung, als auch zum Umfeld.

Das Ratsmitglied steht in Kooperationsbeziehungen zu seinem politischen Umfeld. Der direkte Kontakt zum Bürger ist dem Stadtrat wichtig, weil er sich auch als „Vertreter des kleinen Mannes" gegenüber der Verwaltung versteht. Die lokalen Bezirksbeiräte dienen als weiteres Ohr an der Basis. Mit seiner Partei steht er insbesondere zu Wahlkampfzeiten im engen Kontakt. Interessenvertreter wie Bürgerinitiativen, Gewerkschaften oder Wirtschaft treten an ihn heran, um ihren Anliegen Gehör zu verschaffen. Mit anderen Gemeinden der Region versucht er Koalitionen zu bilden, um gemeinsam Anliegen voranzubringen und von anderen, vergleichbaren Großstädten versucht er zu lernen. Die Presse gibt immer wieder Anstöße für 
Initiativen der Stadträte. In der Verwaltung versorgt die Geschäftsstelle des Gemeinderats die Stadträte mit Informationen und Beschlußvorlagen und nimmt die Anfragen und Anträge der Stadträte entgegen. Die Referate mit den Bürgermeistern als Referatsleitern sind die politischen Ansprechpartner in der Verwaltung. Das Presse- und Informationsamt verbreitet die Beschlüsse in der Öffentlichkeit.

\section{Kommunikation mit der Verwaltung}

Die offizielle Kommunikation der Räte (,Legislative“) mit der Verwaltung (,Exekutive“) ${ }^{1}$ ist stark formalisiert. Die Räte entscheiden aufgrund von Vorlagen der Verwaltung und unterbreiten der Verwaltung von sich aus Anträge. Kommunale Sitzungsdienste dienen im Kern der Verwaltung um Vorlagen zu erstellen, auf Anträge zu reagieren, Sitzungen, in denen sie behandelt werden, vorzubereiten und um Protokolle zu erstellen. Sobald die jeweiligen Unterlagen fertiggestellt sind, werden sie den Räten in Ratsinformationssystemen zur Verfügung gestellt.

\subsection{Kommunale Sitzungsdienste}

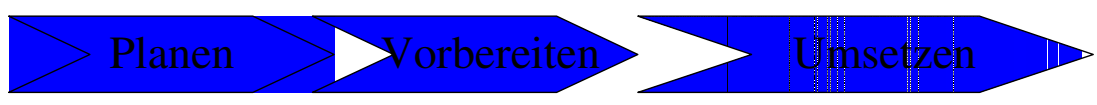

Begleitender Verwaltungsprozess
Die Verwaltung stellt die wichtigsten Informationen für den Gemeinderat zur Verfügung.

Deshalb ist ein kommunaler Sitzungsdienst die wichtigste Voraussetzung für alle weiteren Arten und Komponenten von Ratsinformationssystemen. Typischerweise beinhalten kommunale Sitzungsdienste eine Verwaltung der Sitzungstermine, Sitzungsvor- und -nachbereitung einschließlich Tagesordnung sowie Vorlagen- und Protokollerstellung in der Verwaltung, Anträge, Anfragen von Mitgliedern des Gemeinderats, ein Archiv mit Altbeschlüssen, ein Verfahren zur Sitzungsgeldabrechnung sowie eine Dokumentation zum Ortsrecht (vgl. z.B. (Weinberger 1992, S. 97f)). Wiedervorlagefunktion, Statusübersicht (z.B. für Anfragen, Anträge) und Beschlußkontrolle von Ratsbeschlüssen können ebenfalls in einem Kommunalen Sitzungsdienst integriert sein.

In kleinen Kommunen reicht als Werkzeug des Kommunalen Sitzungsdienstes ein PC im Büro des Bürgermeisters oder des Hauptamtsleiters aus. Auf diesem werden zentral die Gemeinderatsunterlagen (zumindest in der Endfassung) erstellt und digital abgelegt. Mit zunehmender Gemeindegröße werden die Entscheidungen arbeitsteilig vorbereitet; insbesondere an der Vorlagenerstellung ist eine zunehmende Zahl von Akteuren aus der Verwaltung beteiligt. Solange die Zahl von Akteuren überschaubar ist, reichen als kommunaler Sitzungsdienst ein gemeinsamer Arbeitsbereich (z.B. ein gemeinsam verfügbares Verzeichnis auf einem Netzwerkserver) und die Kommunikation über elektronische Post zur gemeinsamen Erstellung der Unterlagen aus. Werden die Prozesse komplexer und intransparenter, dann basieren kommunale Sitzungsdienste auf Workflowsystemen. Darin werden die Prozesse insbesondere zur Vorlagenerstellung und Antragsbearbeitung einmalig festgelegt; die Weiterleitung von einzelnen Entwürfen zwischen den Arbeitsstationen geschieht automatisch und der Status der Vorhaben ist den Verantwortlichen transparent. Da diese Transparenz Handlungsspielraum und Flexibilität der beteiligten Mitarbeiter (und damit möglicherweise auch ihre Macht) einschränkt, erweist sich die Einführung von Workflowsystemen als Kommunale Sitzungssysteme in Kommunen häufig als schwierig.

\footnotetext{
${ }^{1}$ Formal gesehen sind die Räte in Kommunen auch Teil der Exekutive, ihrem Selbstverständnis nach aber häufig Teil der Legislative, weil sie typische Rechte wie die Verabschiedung von Budgets und Gemeindesatzungen haben.
} 
Eine Hauptherausforderung für den produktiven Einsatz von Kommunalen Sitzungsdiensten besteht in der Digitalisierung der benötigten Unterlagen. Zwar werden die meisten per Computer erzeugt, für Zwischenschritte ist es jedoch nach wie vor üblich auf Papierfassungen zurückzugreifen. Sei es, weil wichtige Akteure noch nicht mit Computern ausgestattet sind; sei es, weil in einer Verwaltung mehrere, miteinander nicht kompatible Textverarbeitungssysteme im Einsatz sind oder, weil die einzelnen Ämter noch nicht ausreichend miteinander vernetzt sind. Der Einsatz eines kommunalen Sitzungsdienstes erfordert damit eine leistungsfähige DV-Infrastruktur und eine Standardisierung der Software - zumindest für die Textverarbeitung. Während die einheitliche digitale Verarbeitung von einfachen Textdokumenten technisch gelöst und damit hauptsächlich ein organisatorisches Problem ist, wirft die Verarbeitung von Nichtstandarddokumenten (z.B. Bau- oder Haushaltsplänen) auch technische Probleme auf. Deshalb werden diese Dokumente in der Anfangsphase häufig von der digitalen Verarbeitung ausgenommen, wenn sie nicht ohnehin z.B. in einem geographischen Informationssystem der Verwaltung zur Verfügung stehen. Einzelne externe Dokumente, die in einem gängigen Papierformat vorliegen, können auch eingescannt und dann digital zur Verfügung gestellt werden.

Ein standardisiertes Verfahren zur Bearbeitung von Gemeinderatsunterlagen (z.B. die Kombination der Textverarbeitung Microsoft Word mit der dem Dokumentendatenbanksysteme Lotus Notes) ist in der Regel nicht geeignet zur dauerhaften Archivierung von Gemeinderatsunterlagen, da die Software und die durch sie verwalteten Dokumententypen schnell veralten. Hierdurch gerät die Kommune in eine große Abhängigkeit vom Hersteller. Deshalb gehört zu einem kommunalen Sitzungsdienst ein Archivsystem, welches die Dokumente in einem dauerhaften digitalen Dokumententyp (z.B. ASCII, tif oder pdf) auf relativ dauerhaften Medien (z.B. CD-ROM) speichert.

Seit der Verabschiedung von deutschen Gesetzen und europäischen Richtlinien zur digitalen Signatur ist eine elektronische Unterschrift zwar prinzipiell möglich; es ist aber derzeit noch nicht absehbar, wann die Gesetze und Verwaltungsvorschriften angepaßt werden und entsprechende Software in Verwaltungen Verbreitung findet. Bis dahin kann nur die intern wirkende Mitzeichnung elektronisch abgewickelt werden; für die Rechtmäßigkeit nach außen müssen die erforderlichen Unterschriften meist noch auf Papier erfolgen. Somit ist ein vollständiger Verzicht auf Papierfassungen derzeit noch nicht möglich.

\subsection{Ratsinformationssysteme im engeren Sinne}

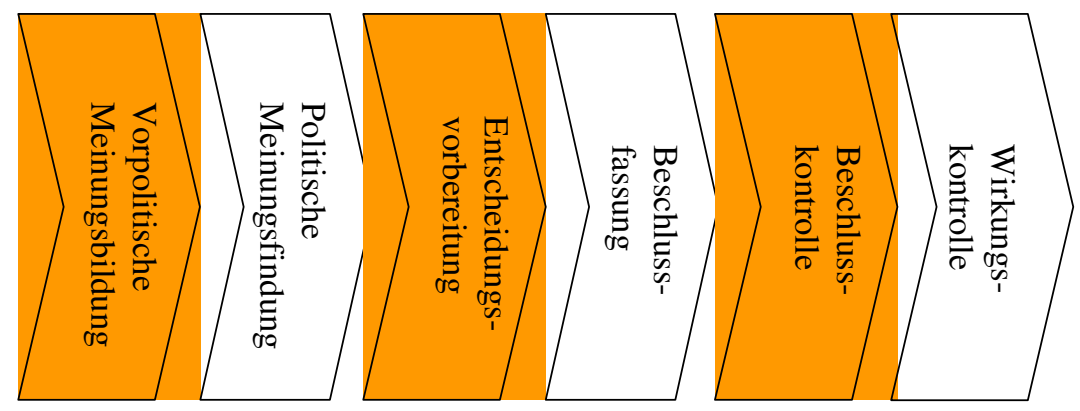

Ratsinformationssysteme (RIS) (Schwabe 2003b) stellen den Gemeinderäten Informationen aus dem Kommunalen Sitzungsdienst zur Verfügung. Sie unterstützen dadurch umfassend die Entscheidungsvorbereitung. Die elektronische Dokumentation von

Beschlüssen unterstützt auch die Beschlusskontrolle, denn jetzt sind Protokolle und Beschlüsse leichter auffindbar (die Informationsflut grosser Kommunen macht eine Papierablage durch jeden einzelnen Rat aussichtslos). Alte Beschlüsse können auch zur vorpolitischen Meinungsbildung dienen, zumindest um Fehlerwiederholungen zu vermeiden oder auf einem unvollständigen Wissen über den Sachstand aufzubauen. 
Im einfachsten Fall stellt die Verwaltung in den Fraktionszimmern PCs auf, mit denen die Räte aktuelle Unterlagen einsehen und im Archiv nach älteren Unterlagen recherchieren können. Die Verwaltung muß dabei jedes Dokument für die Einsicht durch die Gemeinderäte freigeben und dabei auch für den Datenschutz Sorge tragen. Hierbei werfen insbesondere die Freigabe von nichtöffentlichen Vorlagen, Tischvorlagen sowie von Personalangelegenheiten Probleme auf, die auf der Basis von Datenschutzgesetzen, Gemeindeordnung und Hauptsatzung zu lösen sind.

Stehen die primären Gemeinderatsunterlagen - also Vorlagen, Anträge, Tagesordnungen und Protokolle in einem RIS bereit, werden diese häufig durch weitere Unterlagen aus der Verwaltung ergänzt (z.B. Gesetzestexte, Telefonbücher, Ämterverzeichnisse oder Haushaltspläne). Die Informationen eines RIS decken sich zu einem großen Teil mit Informationen, die in den Intranets der Verwaltung für die Verwaltungsmitarbeiter oder in den Bürgerinformationssystemen für die Bürger bereitgestellt werden. Dabei ist allerdings zu beachten, daß die Informationen den unterschiedlichen Interessensgruppen zu unterschiedlichen Zeitpunkten zur Verfügung stehen (meist zuerst der Verwaltung, dann dem Gemeinderat und zuletzt der Öffentlichkeit).

Der Nutzen dieser RIS liegt primär in der schnelleren Verfügbarkeit und dem einfacheren Zugriff auf Informationen und damit (nach einer Anlaufzeit) in einer Entlastung sowohl der Räte, als auch der Verwaltung (Reduktion der Rechencheraufträge) Es besteht die Erwartung, daß die Räte dadurch besser informiert Anträge stellen, Beschlüsse fällen und die Verwaltung kontrollieren. Wesentliche Voraussetzung ist die rechtzeitige und (im Rahmen der gesetzlichen Möglichkeiten) vollständige Vorhaltung aller Unterlagen sowie der flexiblen und benutzerfreundlichen Aufbereitung der Daten in Datenbanken.

Ist in den Fraktionszimmern über die Zugangskontrolle zu den Räumlichkeiten und die direkte Einbindung in das Verwaltungsnetz einigermaßen sichergestellt, daß die Daten aus dem RIS nur von zulässigen Personen für zulässige Zwecke genutzt, ist dies deutlich schwieriger bei Systemen zu gewährleisten, auf die über Telefoneinwählverbindungen oder über das Internet vom heimischen PC oder Notebook aus zugegriffen wird. Hier ist die zulässige Nutzung durch eine sinnvolle Kombination von technischen Schutzmaßnahmen auf Seite der Server und heimischen Rechner sowie von Verpflichtungserklärungen der Gemeinderäte sicherzustellen. Die Herausforderung ist es dabei, nicht lediglich auf ein Höchstmaß an Sicherheit abzuzielen, sondern einen praktikablen Kompromiß zwischen Sicherheitsanforderungen und Nutzbarkeit des Systems durch die Räte zu finden.

Aus wirtschaftlichen Gründen wäre es für die Kommunen attraktiv, das RIS zur Zustellung der Gemeinderatsunterlagen zu verwenden und auf Papierkopien zu verzichten. Dies scheitert heute meist immer noch an den Lesegewohnheiten der Räte, wie auch an den Vorschriften der Gemeindeordnungen und Hauptsatzungen, die eine schriftliche Zustellung vorschreiben. Es ist weiterhin zweifelhaft, ob die digitale Bereitstellung den Anforderungen der Schriftlichkeit genügt und ob die Bereitstellung in einer Datenbank eine Zustellung darstellt. 


\section{Kommunikation innerhalb des Rats}

Weil die Kommunikation in Entscheidungsgremien komplex ist, unterliegt die offizielle Kommunikation im Gemeinderat (zum Teil alten) strengen Regeln, welche die Fairness der

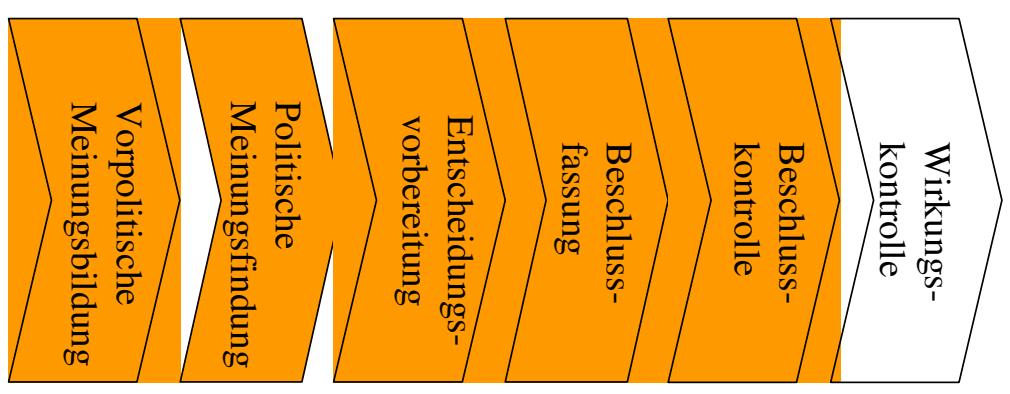
Verfahren sicherstellen sollen. Für die einzelnen Fraktionen gibt es keine vergleichbaren Regeln und entsprechend ,organisch gewachsen" ist hier das Kommunikationsverhalten. Schon einfache Bürosysteme in Verbindung mit E-Mail können hier sehr hilfreich sein. Fortgeschrittene Ratskooperations- und -koordinationssysteme strukturieren die Zusammenarbeit und lassen die einzelnen Fraktionen deutlich handlungsfähiger werden. Davon profitieren insbesondere große Fraktionen. Derartige Systeme unterstützen fast den gesamten Politikprozess mit besonderen Stärken in der politischen Meinungsbildung und der Entscheidungsvorbereitung.

\subsection{Ratskommunikationssysteme und Bürosysteme}

Erst mit der Verbreitung der PCs gewinnen Ratsinformationssysteme an Attraktivität für die Ratsmitglieder. Ähnliche Systeme in Landtagen wurden von den meisten Abgeordneten trotz eines reichhaltigen Informationsangebots - wenig genutzt, weil die Software zu wenig benutzerfreundlich war und weil es sich aus Sicht der einzelnen Abgeordneten nicht lohnte, nur deshalb den Umgang mit dem Computer zu erlernen, um "gelegentlich" zu recherchieren. Anders die Situation seit dem Aufkommen des PCs und des Internets: Hier lohnt es sich schon aus beruflichem und privatem Interesse, den Umgang mit dem PC zu erlernen; ein Ratsinformationssystem wird dann von den Räten als ein integraler Bestandteil eines umfassenden, persönlichen Informationssystems angesehen, wenn es zumindest die gesamte Gemeinderatsarbeit sowie private Korrespondenz umfaßt. Zur Gemeinderatsarbeit gehört in der Regel neben Abruf von Informationen, das eigene Verfassen von Texten (Anträge, Briefe an Bürger...), Recherchen im Internet wie auch zunehmend Korrespondenz mit Bürgern, Verwaltungen, Unternehmen und Parteien via elektronische Post. Eine Verwaltung, die es sich zum Ziel setzt, Gemeinderatsarbeit produktiv und attraktiv zu machen, ist deshalb gut beraten, wenn sie diese Arbeitsweise der Gemeinderäte bei der Gestaltung eines Ratsinformationssystems berücksichtigt. Schon die Möglichkeit für Gemeinderäte, mit Mitarbeitern aus der Verwaltung asynchron via elektronische Post zu kommunizieren, erhöht deutlich die Flexibilität der meist abends oder am Wochenende mit iVorbereitungen beschäftigten Gemeinderäte. Die verbesserte Kommunikationsmöglichkeit unterhöhlt jedoch tendenziell - wie schon die Nutzung des Telefons - die Einhaltung eines Dienstweges.

Die Einbeziehung von PC, elektronischer Post und Internet in ein RIS, werfen eine Reihe neuer Fragen auf. Aus rechtlicher Sicht ist eine Abgrenzung der Gemeinderatssphäre von der Privatsphäre geboten. Die einfachste Lösung dazu, ist die Verwendung verschiedener Geräte für beide Sphären. Dies reduziert die Attraktivität für das Ratsmitglied jedoch erheblich, zumal der Übergang fließend ist (es ist für den Gemeinderat schwer verständlich, warum er die Daten aus seiner Partei oder von seinem Hundezüchterverein auf dem einen Rechner bearbeiten soll und, wenn er diese dann im Gemeinderat verarbeiten will, sie auf einem weiteren Rechner neu erfassen soll). Im Projekt Cuparla (Schwabe, 2000) wurden gute Erfahrungen damit gemacht, zu dulden, daß die Räte die installierte Software (Office-Paket, elektronische 
Post, World Wide Web) auch für private Zwecke nutzen; sie wurden aber verpflichtet, keine neue Software auf den Computern zu installieren (um diese wartbar zu erhalten) und ihr Notebook wurde mit Viren- und Zugriffsschutz abgesichert. Es wurde festgelegt, daß die Software und die Datenbanken des RIS zur Gemeinderatssphäre gehört (und damit auch den Regeln für die DV in der Verwaltung unterliegt); das Dateisystem und die dort gespeicherten Daten hingegen gehörte zu der Privatsphäre des Ratsmitgliedes.

Die Investition in eigene Geräte läßt sich derzeit nur in großen Städten wirtschaftlich rechtfertigen; der Zeitaufwand für Gemeinderatsarbeit zu Hause ist in kleineren Städten bzw. Kommunen zu gering. Gerade kleine Kommunen bietet die Möglichkeit der Co-Nutzung (privat und für Gemeinderatsarbeit) und der daraus folgenden gemeinsamen Finanzierung der Geräte die Chance, mit verhältnismäßig geringem Aufwand ein RIS aufzubauen. Bereits 1998 zeigte sich in einer bei 1'299 Ratsmitgliedern durchgeführten Befragung (vgl. (Schwabe et al. 2000a; Schwabe et al. 2000b)) die überwiegende Mehrheit bereit, mindestens die Hälfte der Gerätekosten zu übernehmen. Diese Bereitschaft überrascht deshalb kaum, weil schon zu jener Zeit viele Ratsmitglieder ihre individuelle Ratsarbeit mit dem privaten PC erledigten.

Liefen noch um 2000 die meisten Systeme zur Unterstützung der Kommunikation innerhalb des Rats auf PCs und Notebooks, setzen sich seit Ende der 90er Jahre Mobiltelefone und PDAs durch. In jüngster Vergangenheit wachsen diese beiden Gerätetypen zu Smartphones zusammen. Diese erreichen eine Leistungsfähigkeit, die es erlaubt, auch für den PC gedachte Anwendungen auf ihnen laufen zu lassen; als prominentestes Beispiel gilt E-Mail. Da Smartphones wirklich mobil sind und zudem mehrere Kommunikationswege (E-Mail, Chat, Instant Messaging, Telefon) in sich vereinigen, kommen sie den Präferenzen der Räte stark entgegen. Es ist deshalb zu erwarten, dass sie sich gerade unter der jüngeren Generation von Politikern in naher Zukunft stark verbreiten.

\subsection{Ratskoordinations- und Ratskooperationssysteme}

Ein Gemeinderat agiert nur selten isoliert; vielmehr ist er in ein Geflecht aus Kooperationsbeziehungen, u.a. mit Fraktionskollegen, Räten anderer Fraktionen, Bürgermeistern und Geschäftsstellen des Gemeinderats sowie der Fraktionen eingebunden. Ratskoordinationssysteme dienen dazu, die Zusammenarbeit dieser Akteure zu koordinieren, indem den Akteuren Werkzeuge zur halbautomatischen Terminvereinbarung, zur Aufgabenverteilung (z.B. über eine gemeinsame to-do-Liste in der Fraktion) und zur Ressourcenverwaltung (z.B. für Räume) in die Hand gegeben werden. Ratskooperationssysteme unterstützen die Gemeinderäte bei ihrer eigenen Initiative, indem sie den Fraktionen gemeinsame elektronische Arbeitsbereiche zum gemeinsamen Verfassen von Dokumenten (z.B. Anträgen) und gemeinsame elektronische Ablagen für fraktionsinterne Unterlagen bereitstellen. Die Erprobung eines Ratskooperations- und -koordinationssystems im Projekt Cuparla in der Landeshauptstadt Stuttgart (vgl. Schwabe 2000) zeigte den Nutzen - aber auch den erheblichen organisatorischen Einführungsaufwand für diese Systeme.

Derzeit ist nicht absehbar, wie die Arbeit der Räte mit einem Workflowsystem unterstützt werden kann, denn die Vorgänge der Ratsarbeit sind zu wenig strukturiert und unterliegen auch einem politischen Diskurs. Wie soll beispielsweise verfahren werden, wenn eine Fraktionsordnung vorschreibt, dass ein Antrag dem Fraktionsvorstand vorgelegt werden muss, bevor er der Verwaltung mitgeteilt wird - und das Gesetz gleichzeitig jedem Ratsmitglied das Recht gibt, Anträge zu stellen? ${ }^{2}$

\footnotetext{
2 Ähnliche Probleme sieht die Kommunale Gemeinschaftsstelle für Verwaltungsvereinfachung KGST (Kassner 2004, S. 20) mit Verweis auf das Beispiel der Stadt Bonn.
} 
Das Cuparla-Projekt wählte einen kontextorientierten Ansatz zur Unterstützung der Kooperation. Abbildung 3 zeigt die Cuparla-Eingangshalle mit den Räumen, die den einzelnen Stadträten zur Verfügung gestellt werden. Jeder Raum hat seine eigene Zugangsberechtigung. "Betritt" ein Stadtrat das virtuelle Arbeitszimmer, befindet er sich in seinem individuellen Arbeitskontext, zu dem nur er Zugang hat. Der Raum "Fraktion" steht für seine eigene Fraktion; die anderen Fraktionen sind für ihn unsichtbar. Zum "Gemeinderat" haben alle Stadträte Zugang, in "Verwaltung" und "Bibliothek" sind alle Dokumente abgelegt, die für Mitarbeiter der Stadt und Stadträte offen sind, usw.

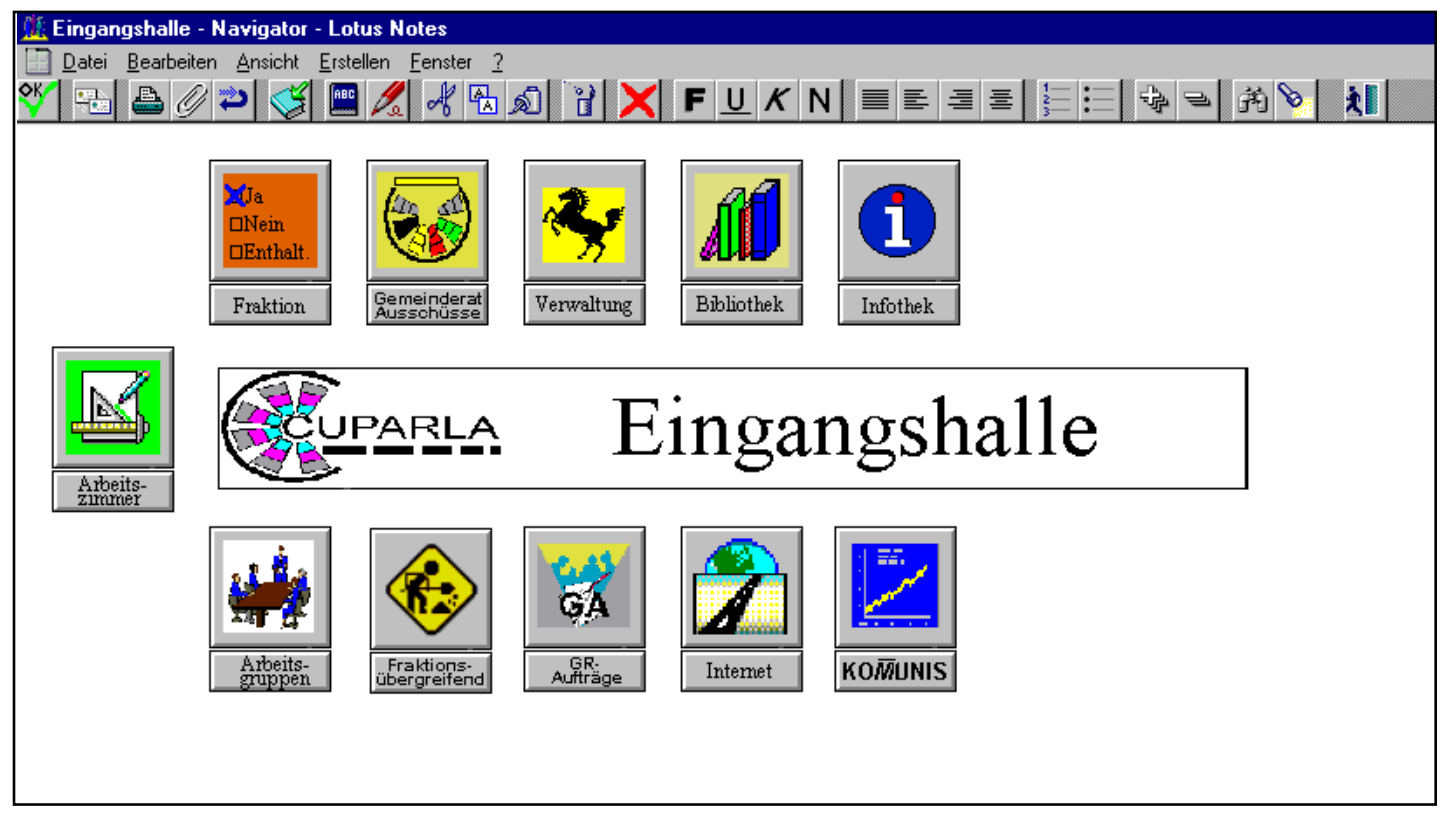

Abbildung 3: Cuparla Eingangshalle

In jedem virtuellen Raum finden die Räte Plätze, an denen sie Dokumente für die eigene oder gemeinsame Arbeit ablegen können, z.B. befindet sich im "Fraktionszimmer" ein "Sitzungstisch", auf dem die Unterlagen für die nächste Fraktionssitzung von jedem Fraktionsmitglied abgelegt und eingesehen werden können. In Laufe der Ratsarbeit werden Dokumente typischerweise zwischen verschiedenen Räumen hin und hergeschoben, um sie jeweils mit einer anderen Gruppe zu diskuiteren (normalerweise wird im privaten Arbeitszimmer begonnen, das Dokument am Ende der internen Diskussion bei der Verwaltung abgelegt und dadurch allen Mitgliedern von Rat und Verwaltung zur Verfügung gestellt).

In internen Sitzungen (z.B. in den Fraktionen) gibt es neben formal organisierten Kommunikationsformen zur Entscheidungsfindung (vgl. Kapitel 5) anderen Formen: Zum einen haben Sitzungen den Informationsaustausch zum Ziel. Für Ihre produktive Durchführung ist eine gute Vorbereitung und die Bereitstellung von Unterlagen (wie im oben genannten CuparlaSystem) wesentlich. Sitzungen können auch das Ziel haben, den inneren Zusammenhalt zu stärken, die Mitglieder zu motivieren und die Gruppe auf ein gemeinsames Ziel auszurichten. Derartige Sitzungen hängen in starkem Masse von Persönlichkeiten ab. Ein dritte Gruppe von Sitzungen hat die gemeinsame Lösung eines wichtigen Problems zum Ziel. Hierfür werden während Klausuren in workshopartiger Atmosphäre gemeinsam Vorschläge und Konzepte erarbeitet. Ein Beispiel für eine solche Sitzung sind die jährlichen Haushaltsklausuren der Fraktionen, in denen die Räte über ihre Budgetprioritäten entscheiden. 
Für diese Art von Sitzungen bieten sich Unterstützungssysteme wie z.B. GroupSystems an . (für eine Beschreibung von Haushaltsklausursitzungen in Stuttgart siehe (Schwabe\&Krcmar 2000b; Klein 2004)). GroupSystems ist eine Software, die der Gruppe Moderationsmaterialien auf dem Computer zur Verfügung stellt. An die Stelle von Papierkarten treten elektronische Kärtchen, an die Stelle von Wandzeitungen große Projektionsflächen. Elektronische Karten können in einem Brainstorming erzeugt, mit einem Voting-Werkzeug bewertet sowie ausgewählt und mit einem Gliederungswerkzeug strukturiert und ausgearbeitet werden.

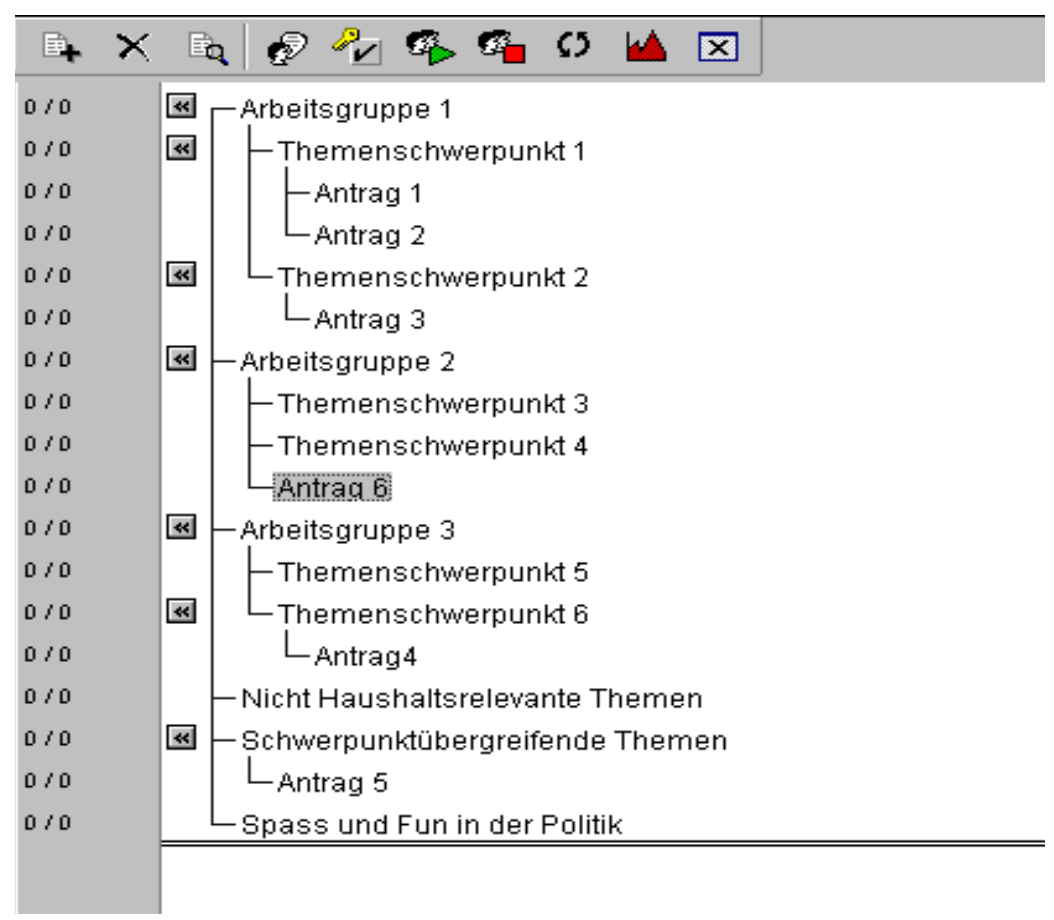

\section{Abbildung 4: Gliederungsentwurf als gemeinsames elektronisches Material auf einer Haushaltsklausursitzung mit GroupSystems (Quelle (Krcmar et al. 2002))}

Abbildung 4 zeigt eine Gliederung von GroupSystems, in der jeweils Teilgruppen für Themenschwerpunkte Anträge entwerfen, um diese dann im Plenum zu diskutieren. GroupSystems erlaubt es in diesem Beispiel nicht nur, dass in der Kleingruppe jedes Mitglied aktiv schreibend mitwirken kann, sondern auch, dass jedes andere Fraktionsmitglied einer Kleingruppe Input geben - oder zumindest sehen kann, wo die anderen Kleingruppen stehen um von deren Arbeiten zu profitieren.

GroupSystems bietet mehrere Vorteile (vgl. z.B. (Schwabe 1995)):

Paralleles Arbeiten: Alle Teilnehmer können gleichzeitig ihre Ideen und Meinungen äußern.

Mehr Beteiligung durch Anonymität: Unabhängig von Macht- und Statusunterschieden können die Teilnehmer in der Sitzung ihre Standpunkte einbringen und vertreten.

Visualisierung: Auf dem Großbildschirm lässt sich für alle lesbar der Stand der Sitzung verfolgen.

Synchrone Sitzungsprotokollierung: Alle erzeugten Ideen und Abstimmungsergebnisse werden automatisch erfasst, lassen sich als Bericht speichern sowie ausdrucken und können in weiteren Sitzungen erneut verwendet werden. Damit ist eine hohe Flexibilität des Arbeitsmaterials gegeben. 
Die Haushaltsklausursitzungen, die in Stuttgart in dieser Form durchgeführt wurden, waren recht erfolgreich. Dennoch hat sich GroupSystems dort nicht dauerhaft etabliert. Es ist schon schwierig, sich von der "natürlichen" Kommunikation, d.h. von den beiden Kommunikationsformen, die jeder Mensch ab Kindheit gelernt, hat zu lösen (Lehrer-Schüler-Verhältnis und "Durcheinanderreden" in der Familie) und mit einer Moderation strukturierte Problemlösungstechniken (Van Gundy 1988) zuzulassen. Der Schritt zur technikunterstützten, moderierten Sitzung ist ein noch grösserer - nicht zuletzt wegen der immer noch hohen Kosten.

\section{Unterstützung der Entscheidungsfindung}

Der größte Teil der politischen Kommunikation findet in Sitzungen statt. Diesen Teil empfinden viele Ratsmitglieder als wenig produktiv, werden doch häufig die klassischen Schaufens-

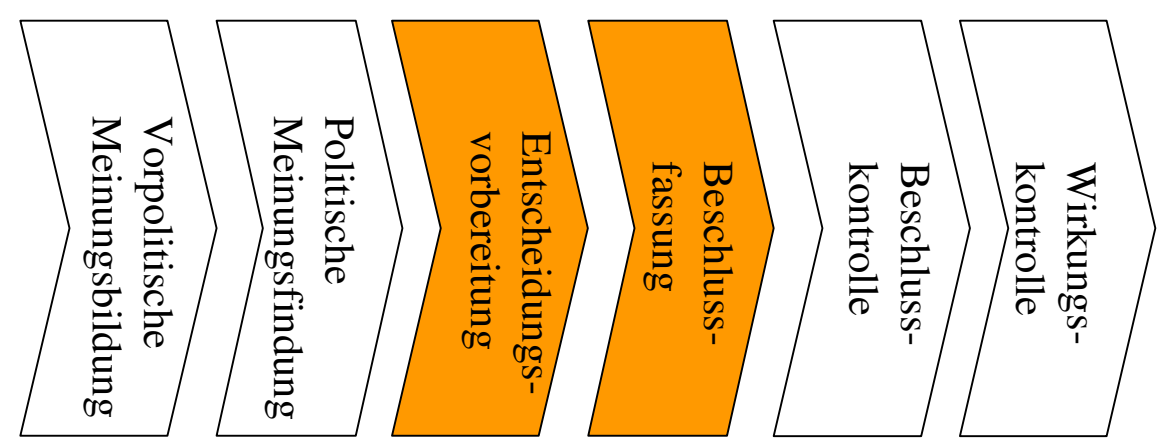
terreden gehalten und kommt der einzelne zu wenig $\mathrm{zu}$ Wort. Abstimmungen dauern besonders lange, wenn sie anonym durchgeführt werden. Für öffentliche Sitzungen kann diesem Problem durch automatische

Sitzungsmanagementsysteme begrenzt begegnet werden. Die KGST (Kassner 2004, S. 25) nennt als Beispiel für den Einsatz eines derartigen Systems die Stadt Gladbeck.

„In der Stadt Gladbeck wird ein IT-gestütztes Sitzungsmanagementsystem eingesetzt, mit dem Rednerliste und Abstimmungen gesteuert werden. Jeder Platz eines Ratsmitgliedes ist mit einer PC-Maus ausgestattet, die jeweils durch eine persönliche Chipkarte aktiviert wird. Damit kann die Anwesenheit eines Ratsmitgliedes ebenso fest gestellt werden wie Wortmeldungen in der tatsächlich abgegebenen Reihenfolge. Abgestimmt wird per Mausklick, wobei das Abstimmungsergebnis über zwei Beamer auf zwei Leinwänden im Ratssaal angezeigt wird. Das Abstimmungsverhalten der einzelnen Fraktionen kann ebenso angezeigt werden wie - bei namentlichen Abstimmungen - die Stimmabgabe jedes Einzelnen.“

Neben dieser Unterstützung des Sitzungs- und Abstimmungsprozesses sind auch die in der Wirtschaft und Wissenschaft diskutieren und verwendeten Entscheidungsunterstützungssysteme (EUS) (Krcmar 1990) verwendbar. Diese EUS aggregieren alle entscheidungsrelevanten Daten in einem Entscheidungsmodell. Mit diesem Modell lassen sich dann die Konsequenzen von Entscheidungen durchspielen. So können Verkehrssimulationen z.B. für die Stadtplanung hilfreich sein oder Modelle der Bevölkerungsentwicklung für die Finanzplanung in der Zeit einer alternden Arbeitsbevölkerung Aufschluss geben. Mit der Entwicklung von Data Warehouses ist derzeit eine Rennaissance der EUS zu beobachten. Die primäre Herausforderung dafür ist die Bereitstellung und Integration aller entscheidungsrelevanten Daten. Werden solche Daten für Entscheidungsträger gezielt und integriert bereitgestellt, ohne ihnen Werkzeuge für die Auswertung zur Verfügung zu stellen, spricht man von Führungsinformationssystemen.

Der IuK-Leiter der Stadt Stuttgart, Andreas Majer, verwendet folgendes Beispiel, um seine Vision eines Führungsinformationssystems zu erläutern (sinngemäß übersetzt aus (Schwabe 2003)): “Stellen Sie sich vor, ein Rat will den Erfolg von Sozialarbeitern in schwierigen Schulen analysieren. Teile der Antwort wird er in den offiziellen Dokumenten finden, die die lokalen Schulen betreffen. Statistische Daten über die Schulen und ihr soziales Umfeld wer- 
den durch das statistische Informationssystem Communis zur Verfügung gestellt und die Finanzdaten zu jeder Schule sind im ERP-System zu finden. Der aktuelle Plan zur Schulentwicklung ist in den Dokumenten aus der Jahresplanung, und die geographische Lage ist in der digitalen Karte des geographischen Informationssystems enthalten. Bisher muss die Information aus vielen Informationssystemen mühsam zusammengesucht werden. Ein Führungsinformationssystem sollte ausreichen, um alle Informationen zusammen abzurufen - und zwar so, dass die Informationen miteinander verbunden sind." Aufbauend auf einem solchen Führungsinformationssystem sind Entscheidungsunterstützungssysteme realisierbar.

\section{Unterstützung der Kontrolle}

Von Gemeinderäten werden die mangelhaften Kontrollmöglichkeiten der Verwaltung beklagt (vgl. Brandel et al. 1998); insbesondere fehlt es den Gemeinderäten an Möglichkeiten zur Auftrags- und Beschlußkontrolle. Ein Ratsauftragsmanagementsystem, wie es z.B. in Wup-

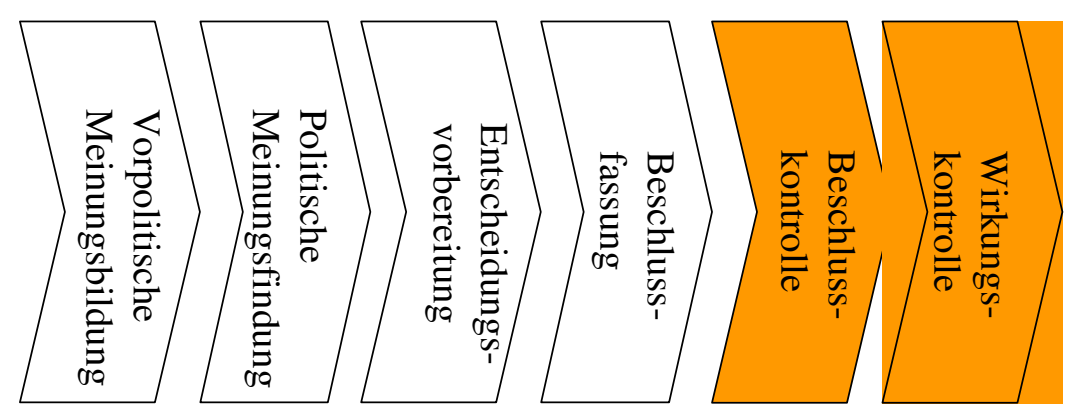
pertal eingeführt wurde, sammelt alle Aufträge aus dem Gemeinderat (insbesondere Beschlüsse, Anträge und Anfragen; aber auch von der Verwaltung zugesagte, regelmäßige Berichte), macht ihren Bearbeitungsstatus für den Rat und die Verwaltungsspitze transparent und erlauben es dadurch, ihre Führungsaufgabe wahrzunehmen. Die Transparenz kann z.B. durch ein Ampelsystem hergestellt werden, indem ein Vorhaben als im grünen Bereich (wie vereinbart in Arbeit), als im gelben Bereich (verzögert) oder als im roten Bereich (überfällig) gekennzeichnet wird. In Stuttgart wurde ein solches System eingeführt. Binnen weniger Wochen nach der Einführung hatte sich die Pendenzenliste deutlich verringert (Schwabe 2003). Bei der Entwicklung der Benutzerschnittstelle zeigte sich, welch überraschende Wirkungen Designentscheidungen haben können. Die Liste der noch offenen Anträge war typischerweise zu lang, um sie jede Woche detailliert durchzugehen. Deshalb zeigte ein kleines Icon für jeden Antrag an, ob er schon durch die Verwaltung bearbeitet worden war. Ein Rat konnte so jede Woche durch die Liste blättern und nach vergessenen Anträgen suchen. Die Verwaltung reagierte schnell und schon bald zeigte das Icon für alle Anträge an, dass sie bearbeitet werden. Diese Bearbeitung bestand allerdings häufig nur aus dem kurzen Text: „Dieser Antrag wird später bearbeitet".

Die verbesserte Möglichkeit zur Kontrolle eines gefällten Beschlusses ist ein wesentlicher Vorteil, welchen ein elektronisches Ratsinformationssystem bietet. So urteilt die KGST: „Das Misstrauen der Politik, die Verwaltung könnte bei der Duchführung von Beschlüssen auf das Vergessen hoffen, kann damit abgebaut werden. In welcher Form über die Durchführung von Beschlüssen informiert wird, ist örtlich zwischen Politik und Verwaltung einvernehmlich festzulegen“" (Kassner 2004, S.34). Einfache Systeme zur Beschlußkontrolle (im wesentlichen Statusanzeigen für Anträge, Anfragen und Beschlüsse) können in das Sitzungsdienstverfahren integriert werden

Die Kontrolle der Verwaltung geht aber über die Beschlusskontrolle hinaus. Wichtig ist nicht nur, ob ein Beschluss umgesetzt wurde, sondern auch, ob er die gewünschten Wirkungen erzielt hat. Hierzu bedarf es eines ausgefeilten Controllingsystems, welches den Räten einen Gesamtüberblick über wesentliche Kenngrößen der Kommune sowie deren Veränderungen 
durch kommunale Initiativen ermöglicht. Diese sind derzeit aber noch Zukunftsmusik. Sobald Informationssysteme zum Verwaltungscontrolling der Verwaltungsspitze zur Verfügung stehen, bietet es sich an, ein Extrakt dieser Systeme den Räten für die Beschlußkontrolle zur Verfügung zu stellen. Dabei ist das Informationssystem so zu gestalten, daß es einerseits einen guten Überblick über die Gesamtsituation der Kommune (z.B. durch eine Darstellung nach dem Balanced Scorecard Modell (Kaplan u. Norton 1996)) als auch die gezielte Einzelkontrolle von Beschlüssen des Gemeinderats ermöglicht.

Der Gemeinderat der Landeshauptstadt Stuttgart hat 2003 auf der Basis einer umfangreichen Vorstudie beschlossen, mit Korvis ein Führungsinformationssystem für Räte und Führungskräfte zu entwickeln und einzuführen (Majer u. Schwabe 2003), welches Führungs- und Kontrollinformationen integriert. Verwaltungsspitze und Gemeinderat sollen durch „Persönliche Portale" bzw. nutzergruppenspezifische Portale gezielt bei ihren Aufgaben unterstützt werden. Neben steuerungsrelevanten Informationen aus der Verwaltung werden aber auch Informationen aus dem Internet sowie persönliche Informationsbereiche wie z.B. Mail, Kalender, To-Do-Listen, etc. integriert werden. Die Ziele und Zielgruppen lassen sich wie folgt zusammenfassen:

- Entscheidungsrelevante Informationen bereitstellen auf der Basis stadtweiter Informationssysteme sowie des Internets zur Verbesserung der Effektivität, Effizienz, Transparenz und Qualität des Verwaltungshandelns

- Präsentation möglichst in einem "Persönlichen Portal", das um weitere Informationen (z.B. Mail, Kalender etc.) ergänzt wird

- Zielgruppen: Führungskräfte der Verwaltung, wie (Bezirks-) Amtsleitungen - jeweils inkl. Stellvertretung- sowie Führungskräfte des Bürgermeisteramts - jeweils ggf. inkl. Vorzimmer, persönlicher Mitarbeiter und Gemeinderat - sowie Fraktionsgeschäftsstellen

Zur Veranschaulichung eines "Persönlichen Portals" dient als Ideenträger die nachfolgende Grafik:": 


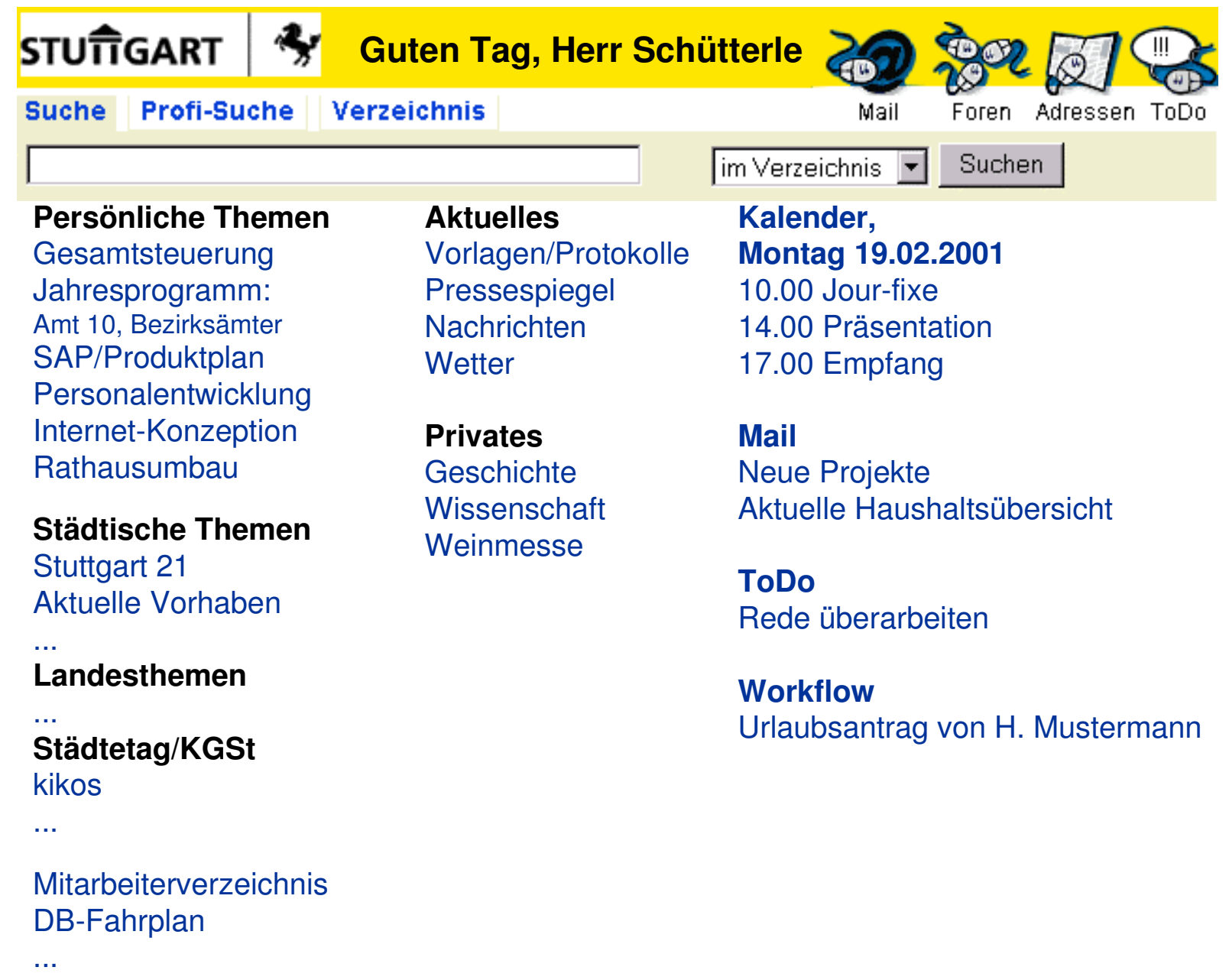

\section{Kommunikation mit dem Bürger}

Da die Politik von den Bürgern gewählt wird, ist eine gute Kommunikation für sie von entscheidender Bedeutung. Das politische System in Deutschland ist (insbesondere im Vergleich zur Schweiz) durch Indirektionen geprägt: Der Bürger fällt wichtige politische Entscheidungen nicht selbst, sondern wählt dafür Abgeordnete . Die politische Meinungsbildung findet zu einem bedeutenden Teil nicht in einem direkten Dialog zwischen Politikern und Bürgern statt,

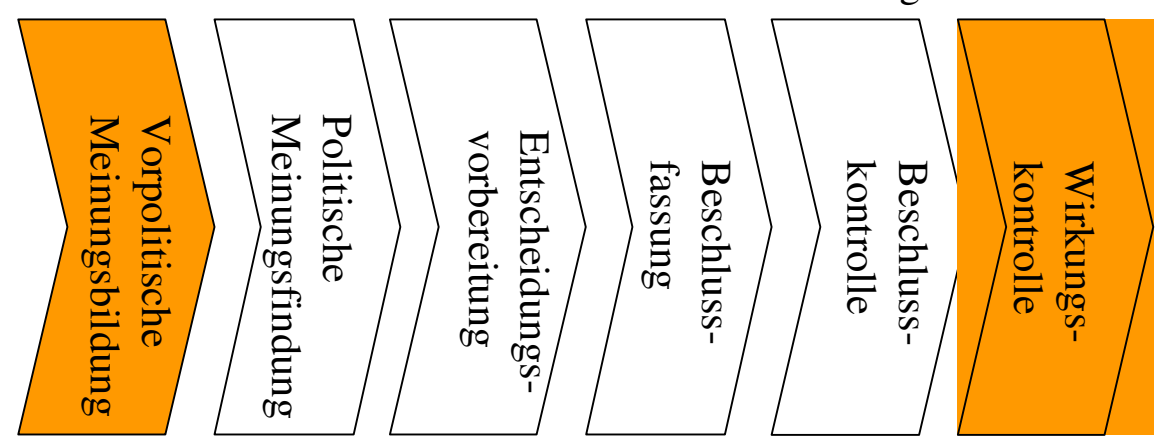

sondern über die Medien (Zeitungen, Talkshows) als Mittler. Deshalb ist der hier nicht weiter vertiefte Umgang mit den Medien für die politische Kommunikation wesentlich. Auch in Deutschland gibt es jedoch direkte Kanäle für die politische Kommunikation zwischen Politikern und Bürgern: 1 . Wenn Politiker (insbesondere bei Wahlen) in Kampagnen die Bürger direkt ansprechen, 2. wenn die Politik (insbesondere die Lokalpolitik) den Bürgern direkt entscheidungsrelevante Information zur Verfügung stellt und ihnen dadurch ermöglicht, sich selbst eine politische Meinung zu bilden und 3. wenn die Politik Bürger gezielt einlädt, am politischen Meinungsbildungsprozess direkt zu partizipieren. In Deutschland dient diese Information und Partizipa- 
tion haupsächlich der vorpolitischen Meinungsbildung und in begrenztem Umfang der Wirkungskontrolle(z.B. wenn den Bürgern Beschwerdemöglichkeiten eingeräumt werden).

\subsection{Bürgerinformation und Kampagnenmanagement}

In vielen Kommunen und erst recht auf Bundes- und Landesebene sind die gewählten Abgeordneten im Internet präsent. Dort präsentieren die Fraktionen ihre Mitglieder, Programme, Pressemitteilungen und Veranstaltungen. Diese Informationen sind über die ganze Legislaturperiode abrufbar und tragen zur Bürgerinformation bei. Bürgerinformation wurde von Steele (1997) definiert als:

- Information über die bürgerlichen, sozialen und politischen Rechte und Ansprüche eines Bürgers sowie über seinen Schutz durch den Staat;

- Information, um bürgerliche, soziale und politische Aspekte des Staates kritisch zu beurteilen und über die Möglichkeit, einen Mißstand zu beheben;

- Information über die bürgerlichen, sozialen und politischen Pflichten eines Bürgers.

Den grössten Teil der Bürgerinformation stellt die Verwaltung einer Kommune, eines Landes oder des Bundes zur Verfügung, deshalb soll in diesem Beitrag nicht weiter darauf eingegangen werden. Die gewählten Volksvertreter können aber selbst zur besseren Bürgerinformation beitragen, indem sie ihre Meinungen publizieren und an elektronischen Diskussionen teilnehmen (per Community oder Chat); sie können auch ihre E-Mail-Adresse publizieren, um dadurch persönlich für Fragen oder bei Problemen zur Verfügung zu stehen. Mit diesem Instrument erfahren sie konkret, wo den Bürger der Schuh drückt - ein Bedarf, den gerade die Lokalpolitik hat, kann sie sich doch durch konkrete Massnahmen besser profilieren, als durch allgemeine politische Stellungnahmen

Der politische Einfluss einer Partei hängt aber nicht nur von der dauerhaften Präsenz ihrer Abgeordneten ab, sondern auch davon, wie schnell es ihr gelingt, ihre Vertreter und Mitglieder zu mobilisieren, um in einer Kampagne gemeinsam und abgestimmt ein Thema in der Öffentlichkeit zu platzieren. Die Kampagnenfähigkeit der Parteien ist durch Intranets (d.h. partei-interne Webseiten) deutlich erhöht worden, ist es ihnen doch jetzt möglich, ihre Mitglieder sehr viel schneller und umfangreicher mit Informationen (z.B. Argumentationshilfen) zu versorgen.

Im Ausland sind schon politische Kampagnen zu beobachten, die mit deutlich schnelleren Medien als mit dem Internet koordiniert werden. Dauert es typischerweise noch mehrere Stunden bis Tage, bis eine Mehrzahl aller Addressaten eine Massen-Email gelesen hat, werden SMS in der Regel binnen weniger Minuten gelesen. Rheingold (2002) berichtet, dass sich bei der politischen Wende in den Phillipen ab 1998 die Demonstranten (sog. „Smartmobs“) über SMS koordiniert haben mit Nachrichten wie „Treffpunkt am Bahnhof, alle Schwarz tragen". Auch die Polizei in der Schweiz berichtet, dass bei den Demonstrationen zum World Economic Forum die Demonstranten kurzfristig die örtlichen Schwerpunkte ihre Aktivitäten über SMS festgelegt haben.

Elektronische Medien führen nicht nur zu einer Beschleunigung der Information und der Adhoc Koordination, sondern verbessern auch grundsätzlich die Koordinationsfähigkeit der politischen Akteure, indem sie ihnen „gemeinsames Material“ (quote Diss) zur Koordination zur Verfügung stellen. Das Potential von gemeinsamem Material als Koordinationsinstrument läßt sich am besten an einem Beispiel erklären. Kollock (1998) dokumentierte die Koordination des 'Schulen-ans-Netz-Tages 1996' in Kalifornien (NetDay 1998). Kaliforniens Schulen waren 1996 schlecht mit Computertechnologie ausgestattet; insbesondere fehlte vielen Schulen ein Anschluß ans Internet. Da auch den Mitarbeitern der kalifornischen 
Computerfirmen die Qualifikation der Schüler ein Anliegen war, bildete sich eine Bürgerinitiative, die sich zum Ziel setzte, möglichst viele Schulen an das Internet anzuschließen. Die Bürgerinitiative wählte den 9. März 1996 zum "Schulen-ans-Netz-Tag" aus. Die Aufgabe der Organisatoren war es nun sicherzustellen, daß möglichst viele Personen teilnahmen, daß sich das Personal gleichmäßig auf die Schulen verteilte und daß die benötigten Ressourcen und das benötigte Wissen in jeder anzuschließenden Schule vorhanden war. Die Initiatoren koordinierten den "Schulen-ans-Netz-Tag" vollständig via Internet. Die einzige Telefonnummer der Aktion verwies via Tonband auf eine Internetseite.

Auf der Internet-Eingangsseite von "Schulen-ans-Netz" war die Karte von Kalifornien abgebildet. Ein Interessent musste sich eine Stadt aussuchen und kam in der Folge auf immer detaillierteren Landkarten bis zur unterster Ebene, wo eine Schule ausgewählt werden konnte. Jede Schule zeigte farbig auf, wieviele Freiwillige sich bereits dafür eingetragen hatten, eine Schule an das Internet anzuschließen. Eine rote Markierung stand für viel zu wenige Freiwillige, eine gelbe für eine kanppe Beteiligung und eine grüne Markierung zeigte an, daß sich für diese Schule schon ausreichend Freiwillige gemeldet hatten. Jede/r Freiwillige konnte sich so gezielt eine Schule aussuchen, in der noch Bedarf zur Mithilfe bestand. Gleichzeitig vermittelten die vielen roten, gelben und grünen Punkte den Beteiligten sowohl den Eindruck benötigt zu werden, als auch das Gefühl, Teil einer großen Bewegung zu sein. Da mit der Ortskarte im Internet auch die Anleitung bereitgestellt wurde, wie man über das Telefon Computer verkabelt und an das Internet anschließt sowie welche Ressourcen dazu benötigt werden, konnte jedes für eine Schule verantwortliche Team seine Arbeit selbst koordinieren. Eine klassische Koordination durch Menschen in Hierarchien entfiel komplett (Kollok 1998).

\subsection{Bürgerpartizipation}

Systeme zur politischen Bürgerpartizipation laden den Bürger zur Beteiligung an politischen Entscheidungsprozessen des Rates ein (vgl. z.B. (Kubicek et al. 2003)). Hierzu wurden (insbesondere in den USA) Erfahrungen mit Hilfe des Internets gesammelt (Schuler 1996). Insbesondere im Agenda 21 Prozeß ist es üblich geworden, Arbeitskreise mit Bürgern zur langfristigen Konzeptentwicklung einzurichten. Diese können wiederum von moderierten Arbeitsformen profitieren. In Deutschland wurde schon seit den 70er Jahren (Daniel 2002) propagiert, die Bürger in die Kommunalplanung einzubeziehen. Damals war aber die Verwaltung die treibende Kraft, d.h. die Bürger wurden in die verwaltungsinternen Planungsprozesse einbezogen. Im Rahmen des Agenda 21 Prozesses wurde auch von der Politik versucht, Bürger direkt in die Planung ihrer Stadtentwicklung einzubeziehen, wie das folgende Beispiel (Schenk u. Schwabe 2000) zeigt. Als eine Vorstufe für den Agenda 21 Prozess wollte die grüne Fraktion des Stuttgarter Gemeinderats im Rahmen einer Zukunftskonferenz die Bürger an der Entwicklung eines Leitbilds für die Gesundheitspolitik beteiligen. Die Zukunftskonferenz wurde von zwei professionellen Moderatoren vorbereitet und mit GroupSystems (siehe Kapitel 4.2) unterstützt.

Als Pilotstudie für den Stuttgarter Agenda 21 Prozess luden Stadträte der "Grünen Bürger" aus allen Schichten ein, um gemeinsam ein Leitbild für eine "gesunde Stadt Stuttgart" zu erarbeiten. Insgesamt wurden 60 Bürger für eine dreitägige Zukunftskonferenz eingeladen.

Der Teilnehmerkreis wurde entsprechend dem Thema „Gesunde Stadt - Stuttgart - Gegenwart und Zukunft" aus mehreren Bezugsgruppen gebildet und Personen eingeladen. Beispielsweise wurden Vertreter von Kliniken, Stadtplanungsamt, Kindergärten, Altenbetreuung etc. angeschrieben. Denn für die Durchführung eines Workshops sollten folgende Tischgruppen gebildet werden:

- Gesellschaft (Politik und Verbände), Finanzierung des Gesundheitswesens 
- Bürgerinitiativen, Patienten und Selbsthilfegruppen

- Pflegende und heilende Berufe und Institutionen

- Freizeit, Sport, Prävention, Erziehung, kultureller Überbau, seelische Gesundheit

- Soziale Realität, Randgruppen

- Versorgung, Sicherheit und Aufsicht

- Planung/Beratung, Verwaltung, Umwelt

Durch den Einsatz von GroupSystems war es möglich, eine grössere Gruppe arbeitsfähig zu halten, als dies bei klassischen Moderationsmaterialien der Fall gewesen wäre. Auf diese wurde hingegen dort nicht verzichtet, wo es für eine gute Visualisierung wesentlich war. Die Teilnehmer profitierten von einem schnelleren Feedback, da alle Arbeitsmaterialien aller Teilgruppen jederzeit für jedermann sichtbar waren. Insgesamt wurde der Sitzungsprozess beschleunigt, da GroupSystems automatisch dokumentierte. Die Teilnehmer hatten viel Spass am Prozess und das Ergebnis war nach Aussagen der veranstaltenden Räte umfassender, als es bei einem traditionellen Prozess war. Negativ wurden die hohen Kosten durch die bereitgestellten Notebooks und Software bemerkt.

Im Vergleich zu Internetforen als Form der Bürgerbeteiligung haben face-to-face Zukunftskonferenzen einen immensen Vorteil. Internetforen unterstützen eher die Bequemlichkeit der Bürger und fordern nicht zu aktivem Engagement bei der Umsetzung der Ergebnisse heraus. Anders die Zukunftskonferenz: Sie bringt Bürger zusammen und ermöglicht es diesen spürbar zu erleben, dass sie mit ihren Interessen nicht alleine stehen. Der Aufbau von Beziehung wird möglich und durch den intensiven Austausch sowie die enge Zusammenarbeit gefördert. Geweckte Begeisterung springt wie ein Funke von einem zum anderen über. Bleiben die Ergebnisse nicht in der Umsetzung stecken bzw. werden sie nicht durch die Politik ignoriert, kann dies ein Mittel sein, der Politikverdrossenheit entgegen zu wirken. Dieser Beitrag zeigte auf, dass die Entscheidung für einen Workshop nicht gleichzeitig eine Entscheidung gegen den Einsatz von Technologie ist; vielmehr bietet sich gerade die Zusammenarbeit in einem Raum für die Technikunterstützung an. Die Vorteile eines Rechnereinsatzes kommen gerade bei Großgruppen zum Tragen. Die damit verbundenen Einschränkungen einer partizipativen Zusammenarbeit können durch diese weitgehend ausgeglichen werden.

\section{Ausblick}

In diesem Beitrag wurde vorgestellt, wie die einzelnen Schritte des Politikprozesses durch IT unterstützt werden können. Wirklich weit verbreitet sind nur die Internetpräsenz einer Kommune sowie einfache Ratsinformationssysteme und damit die Entscheidungsvorbereitung sowie die Selbstvermarktung der Politik und die dadurch mögliche Beeinflussung der vorpolitischen Meinungsbildung. Durch die Parteien wurden Systeme für das Kampagnemanagement vorangetrieben. Eine weitere Verbreitung fortgeschrittener Systeme scheitert häufig am Geld: Die Kosten sind häufig direkt lokalisierbar, der Nutzen bleibt aber diffus der Allgemeinheit zugeordnet. Zwei Trends sprechen für eine beschleunigte Verbreitung der hier angesprochenen Systeme zur Unterstützung der politischen Kommunikation. Den grossen Kommunen ist bewusst geworden, dass die Ratsarbeit und damit auch deren Unterstützung professionalisiert werden muss, will die Kommune den auf sie zukommenden Herausforderungen gerecht werden. In kleineren Kommunen erleichtern leistungsfähig Systeme die Vernetzung Ratsarbeit mit Haupterwerb/Familie. Damit gelten sie als eine Voraussetzung für die Rekrutierung jüngerer Ratsmitglieder und damit für den dringend notwendig gewordenen Generationswechsel.

Häufig unterschätzt wird das Potential, welches die Unterstützung der politischen Kommunikation für die Reorganisation der Verwaltung bedeutet. Wenn die Politik und die Bürger 
ernster genommen werden sollen, ergibt sich fast zwangsläufig ein Bedarf an schnelleren, flexibleren und transparenteren Verwaltungsprozessen.

Während die Kommunen noch damit kämpfen, mit den Herausforderungen und Potentialen des Internets fertig zu werden, steht mit den mobilen Geräten bereits die nächste Technologiegeneration vor der Haustür. Politik wird dadurch für alle Akteure noch allgegenwärtiger und unmittelbarer. Auch verändert sich der Charakter der durch sie übermittelten Information. Sollte sie bisher zur politische Reflektion und Entscheidung beitragen, ermöglicht sie jetzt Aktion und spontane Partizipation. Sie kommt dadurch dem Lebensgefühl der nachwachsenden Generation entgegen, stellt aber auch die derzeit etablierten politischen Kommunikationsstrukturen vor grosse Herausforderungen.

\section{Literatur}

Brandel R; Rusche S, Stöbe-Blossey S, Wohlfahrt N (1998) Verwaltungsmodernisierung und die Entwicklung der kommunalpolitischen Arbeit - Ergebnisse einer Befragung. Projektbericht des Instituts für Arbeit und Technik 1998-06, Wissenschaftszentrum NordrheinWestfalen, Gelsenkirchen.

Daniel P (2002) Die Planungszelle. Der Bürger als Chance. VS Verlag für Sozialwissenschaften

Kaplan R, Norton D (1996) The Balanced Scorecard. Harvard Business School Press, Cambridge

Kassner U (2004) Vom Ratsinformations- system (RIS) zum Ratsportal. Bericht Nr. 4/2004 der Kommunalen Gemeinschaftsstelle

Klein A (2004) Adoption von Electronic Meeting Systems. Gabler, Wiesbaden

Kollock P (1998). The Economies of online cooperation: Gifts and public goods in cyberspace. In: Smith M, Kollock P Communities in Cyberspace. Routledge, London

Krcmar H (1990) Entscheidungsunterstützungssysteme - Hilfsmittel und Werkzeuge. In: Kurbel K, Strunz H, Handbuch Wirtschaftsinformatik. Schäffer-Poeschel, Stuttgart

Krcmar H, Schwabe G, Klein A (2002) Fünf Jahre Telekooperation im Gemeinderat. In: Schuster W, Murawski K, Die regierbare Stadt. Kohlhammer, Stuttgart

Kubicek H, Westholm H, Wind M (2003): Stand und Perspektiven der Bürgerbeteiligung via Internet. Von verstreuten Einzelangeboten zur mandantenfähigen Beteiligungsplattform In: Verwaltung und Management, 9. Jg., H. 2, S. 62-71

Majer A, Schwabe G (2003) Korvis - ein kommunales Rats- und Verwaltungsinformationssystem. In: Beyer L, Frick D, Gadatsch A, Macher I, Paul H (2003) Vom E-Business zur E-Society - New Economy im Wandel. Hampp Verlag, München et al. S. 145-156

Lenk K, Brüggemeier M, Hehmann M, Willms W (1990) Bürgerinformationssysteme. Strategien zur Steigerung der Verwaltungstransparenz und der Partizipation der Bürger. Westdeutscher Verlag, Opladen

NetDay (1998). What's Netday? In: http://www.netday96.com, zugegriffen am 23.06.1998.

Rheingold H (2002) Smart Mobs: The Next Social Revolution. Perseus Books Group

Schenk B, Schwabe G (2000) Die elektronische Zukunftskonferenz. In: Lüttich H, Rautenstrauch C, Verwaltungsinformatik 2000, MDV Halle 2000, S.324-340

Schuler D (1996) New community networks - wired for change. ACM Press New York 
Schwabe G (1996) Die Rolle neuer Informations- und Kommunikationstechnologie für die Bürgerinformation. In: IM - Information Management, Vol. 11, Nr. 2, 1996 S. 6-15

Schwabe G (1997) Germany. In: Steele J, Information for citizenship in Europe. Policy Studies Institute, London 1997, S. 38-107

Schwabe G (1995) Objekte der Gruppenarbeit - ein Konzept für das Computer Aided Team. Gabler, Wiesbaden

Schwabe G (2000) Telekooperation für den Gemeinderat. Kohlhammer, Stuttgart

Schwabe G (2000b) Von Cuparla zu E-Councils: Anforderungen, Erfahrungen, Konzepte. In: Reinermann H, Regieren und Verwalten im Informationszeitalter, Tagung der DHVSpeyer und der Gesellschaft für Informatik; 68. Staatswissenschaftliche Fortbildungstagung vom 15.-17. Maerz 2000; Hüthig Verlag 2000b, S. 444-478

Schwabe G (2003) Growing an Application from Collaboration to Management Support. In: Laudon K, Laudon J, Management of Information Systems. 5. Auflage, Prentice Hall

Schwabe G (2003) Ratsinformationssysteme; Basisstichwort. In: Eichhorn P, Friedrich P, Jann W, Oechsler W, Püttner G, Reinermann H, Verwaltungslexikon, 3. Auflage Nomos

Schwabe G, Falkenstein F, Krcmar H (1997) Bürgerinformation an der Schwelle zur Informationsgesellschaft. In: Verwaltung und Management, Nr. 5, 1997, S.276-281

Schwabe G, Krcmar H (2000) Digital material in a political work context - the case of Cuparla. In: Proceedings of the 8th European Conference on Information Systems ECIS 2000, Wirtschaftsuniversität Wien, S. 1152-1159

Schwabe G, Krcmar H (2000b) Electronic Meeting Support for Councils. In: AI and Society Vol. 14, S. 48-70

Schwabe G, Krcmar H (1998) Sitzungsunterstützung für die Politik. In: Herrmann T, JustHahn K, Groupware und organisatorische Innovation. Tagungsband der Deutschen Computer Supported Cooperative Work - DCSCW '98, Teubner Stuttgart, Leipzig 1998, S. $167-180$

Schwabe G, Vöhringer B, Krcmar H (2000a) Die Arbeitsituation von Gemeinderäten und Möglichkeiten zu ihrer Computerunterstützung - Ergebnisse einer empirischen Untersuchung. Teil 1 In: Verwaltung und Management, Vol. 6, Nr. 4, S. 217-221

Schwabe G, Vöhringer B, Krcmar H (2000b) Die Arbeitsituation von Gemeinderäten und Möglichkeiten zu ihrer Computerunterstützung - Ergebnisse einer empirischen Untersuchung. Teil 2 in: Verwaltung und Management, Vol. 6, Nr. 5, S. 312-315

Steele J (1997) Information for citizenship in Europe. Policy Studies Institute, London

Van Gundy A (1988) Techniques of structured problem solving. 2. Auflage, Van Nostrand Reinhold, New York

Weinberger B (1992) Informations- und Kommunikationstechniken in der kommunalen Praxis - Handbuch für Rat und Verwaltung. 2. Aufl., Deutscher Gemeindeverlag, Köln 